\title{
EDITORIAL
}

\section{Two branches intertwined}

\author{
This Focus issue on plant-microbe interactions showcases advances in our understanding \\ of the intricate relationships between plants and their microbial friends and foes.
}

The global population is predicted to exceed nine billion by 2050, putting unprecedented pressure on already scarce natural resources. To cope with the population increase and the associated increase in food consumption, there is an urgent need not only to optimize food production but also to do so in a sustainable manner. Ensuring long-term global food security is a highly complex issue when considering the projected population increase alone; throw the effects of climate change into the mix and the task seems positively Herculean. Plant and agricultural sciences and associated disciplines will therefore be key areas of development in the future. Much has been written about the need to develop new farming strategies and to invest heavily in agricultural biotechnology. However, one important pressure on global food security that is often overlooked is the threat posed by plant pathogens.

There are many examples of bacterial, fungal, oomycete and viral pathogens that cause devastating losses to staple crops, including rice and wheat, each year. One of the most notorious plant pathogens is the oomycete Phytophthora infestans, the causative agent of the Irish potato famine in the 1840 s, which killed more than one million people. Clearly not a disease of the past, Phytophthora spp. infections are estimated to cost more than US $\$ 6$ billion in damages and infection control efforts every year ${ }^{1}$. P. infestans and the related Phytophthora sojae, which causes soybean root rot, feature in the Review by Pumplin and Voinnet on page 745 and the Review by Wirthmueller, Maqbool and Banfield on page 761.

As highlighted by Hanley-Bowdoin and colleagues on page 777 , geminivirus diseases have also been a major cause for concern over the past 20 years. One of the most prominent agricultural diseases caused by these viruses is cassava mosaic disease. Cassava is part of the daily diet of more than 500 million people in Africa and it is the world's third most important source of dietary energy after maize and rice. Already an important cash crop for millions of subsistence farmers, the reliance of the developing world on cassava is expected to increase in the future, as it is insensitive to drought and tolerant of nutritionally poor soils. The annual losses as the result of the ongoing cassava mosaic disease epidemic in East Africa are estimated to exceed US\$2 billion ${ }^{2}$.

Perhaps chief among the agriculturally important fungal pathogens is the filamentous ascomycete
Magnaporthe oryzae. More than 50\% of the world's population rely on rice as their daily source of energy and M. oryzae is the most common causative agent of rice blast disease, resulting in the loss of $30 \%$ of the rice harvest every year. Moreover, a new strain of $M$. oryzae that causes wheat blast disease has emerged in South America, resulting in yield losses of up to $100 \%$. M. oryzae is one of the many eukaryotic filamentous phytopathogens discussed by Giraldo and Valent on page 800 .

As alluded to above, most of the Reviews in this Focus issue draw on examples from a wide range of plant pathogens. Although the theme of each Review is different, the common thread is the illustration of how new research developments, including advances in structural biology and in planta analyses of effector translocation, are facilitating our understanding of the coevolutionary 'arms race' between plants and their pathogens. The issue also includes a Review on the microbial ecology of the rhizosphere by Philippot and colleagues on page 789, in which they call for an integrative approach that involves both agricultural and natural ecosystems to improve the stability of crop production.

There are of course, by definition, two sides to any plant-microbe interaction. As microbiologists we tend to focus on the latter but there is a compelling need to also support those more interested in the former. In a recent audit of plant pathology training and education in the United Kingdom ${ }^{3}$, the British Society for Plant Pathology (BSPP) highlighted grave concerns about the future of plant pathology in the United Kingdom. The potential problems include the dearth of practical training and the increasing age profile of the remaining plant pathologists in higher education. Ironically, the release of the report in 2012 coincided with the first reports of ash dieback in the United Kingdom, which emphasized the urgent need for increased investment in plant pathology in this country.

In putting together this Focus issue, we hope that it will reach out to microbiologists and plant scientists alike; to safeguard our future food resource demands, it is essential that both branches of research are seen as symbiotic partners, which should be reflected in investment in both research areas.

\footnotetext{
. Haverkort, A. J. et al. Potato Res. 51, 47-57 (2008)

2. Patil, B. L. \& Fauquet, C. M. Mol. Plant Pathol. 10, 685-701 (2009).

3. British Society for Plant Pathology [online], http://www.bspp.org.uk/ society/docs/BSPP\%20plant\%20pathology\%20audit\%202012.pdf (2012).
} 